\section{The long-term result of slanted medial rectus resection in exotropia of the convergence insufficiency type}

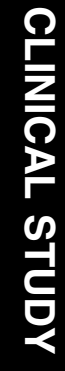

MY Choi ${ }^{1,2}$ and J-M Hwang ${ }^{3,4}$

\section{Abstract}

Purpose To evaluate the long-term results of slanted medial rectus (MR) resection for intermittent exotropia $(X(T))$ of the convergence insufficiency type. Methods In all, 10 patients with an $\mathrm{X}(\mathrm{T})$ greater at near than at distance by 10 prism diopters (PD) or more were included in this prospective study. Patients received slanted bilateral MR resection. The upper edge of the MR was resected according to the distance exodeviation and the lower edge of the MR was resected according to near exodeviation. The postoperative follow-up period was between 6 and 62 months with a mean of 38.9 months. The paired $t$-test was used to compare: mean distance angle of deviation preoperatively and postoperatively; mean near angle of deviation preoperatively and postoperatively; and mean near-distance exodeviation difference preoperatively and postoperatively.

Results Bilateral slanted MR resections reduced mean exodeviation at distance from $23.0 \pm 7.2$ to $16.3 \pm 5.4$ PD $(P=0.03)$; mean exodeviation at near from $34.3 \pm 7.7$ to $24.6 \pm 6.9 P D(P=0.01) ;$ and mean near-distance difference from $11.4 \pm 2.6$ to $8.3 \pm 3.5 \mathrm{PD}$ $(P=0.04)$. At the final follow-up examination, all patients demonstrated an exodeviation of 10 PD or more at distance and near, and the exodeviation difference between distance and near deviation was within 10 PD in five of the 10 patients. Three patients had an esodeviation at distance after surgery, but all resolved within 4 weeks.

Conclusions Bilateral slanted MR resections in patients with $X(T)$ of the convergence insufficiency type resulted in undercorrection in all patients.
Eye (2006) 20, 1279-1283. doi:10.1038/sj.eye.6702095; published online 9 September 2005

Keywords: medial rectus resection; convergence insufficiency; diplopia; exotropia; slanted surgery

\section{Introduction}

The convergence insufficiency type of intermittent exotropia $(X(T))$ is characterized by a greater exodeviation measured at near than at distance of usually 10 prism diopters (PD) or more. ${ }^{1-4}$ Reported symptoms include headache, diplopia, asthenopia, and reading problems. ${ }^{5}$ In mild cases, symptom may be amenable to nonsurgical means such as orthoptic treatment, prisms, plus lens, or psychotherapy. ${ }^{5,6}$ However, some patients with constant deviation, that is, too large at distance or at near, do not respond to any combination of nonsurgical treatments, and may require surgical treatment to relieve symptoms. ${ }^{7}$

The surgical procedures recommended for this form of $\mathrm{X}(\mathrm{T})$ include bilateral lateral rectus (LR) recession using a slanting procedure, bilateral medial rectus (MR) resection using a slanting procedure, or MR resection(s) with an adjustable suture. ${ }^{1,2,7-12}$ Surgical success rates have been reported to vary between 18 and 92\%. ${ }^{1,2,7-12}$ Nemet and Stolovitch ${ }^{8}$ adopted slanted procedures for bilateral MR resection in three patients. Slanted MR resection shortens and tautens MR muscle lower fibres for near vision, and good surgical results have been reported over a year of follow-up. ${ }^{8}$ Biedner ${ }^{9}$ also performed single slanted MR resection in three patients with convergence insufficiency. All patients showed a postoperative alignment within 10 PD in all field gazes without diplopia.
${ }^{1}$ Department of Ophthalmology, Chungbuk National University College of Medicine, Cheongju, Korea

${ }^{2}$ Chungbuk National University Medical Research Institute, Cheongju, Korea

${ }^{3}$ Department of Ophthalmology, Seoul National University College of Medicine, Seongnam, Korea

${ }^{4}$ Seoul National University Bundang Hospital, Seongnam, Korea

Correspondence: MY Choi, Department of

Ophthalmology,

Chungbuk National University

College of Medicine,

Chungbuk National University Hospital, \#62 Kaeshin-Dong, Heungduk-Gu, Cheongju, Chungbuk 361-711, Korea Tel: + 82432696335 ; Fax: + 82432645263

E-mail:mychoi@

chungbuk.ac.kr

Received: 27 April 2005 Accepted in revised form: 3 August 2005

Published online:

9 September 2005

The authors have no financial interest in any of the materials or methods mentioned 
However, they only included three patients in each study, and five of their six patients had low degree of preoperative exodeviation, that is, within $10 \mathrm{PD}$ at distance. ${ }^{8,9}$ No other prospective study has evaluated the surgical success rates of slanted MR resection for $X(T)$ of the convergence insufficiency type. Thus, we undertook this prospective study to evaluate the long-term treatment results of slanted resection of bilateral MR in $X(T)$ with convergence insufficiency.

\section{Methods}

In this prospective study, we treated 10 patients for $X(T)$ of the convergence insufficiency type. All patients had an $\mathrm{X}(\mathrm{T})$ greater at near than at distance by $10 \mathrm{PD}$ or more, and this had been confirmed by repeated examinations at intervals of at least 1 month. No patient with previous ocular or extraocular muscle surgery, ocular or neurologic pathologic conditions, amblyopia, or a postoperative follow-up of $<6$ months was included in this study.

The patients ranged in age from 5 to 17 years (mean, 10.3 years), and the mean length of follow-up was 38.9 months (range, 12-62 months). The symptoms of convergence insufficiency type $X(T)$ were cosmetic in nine cases and diplopia in one (Case No. 6). Push-up training was attempted to build convergence fusional amplitude, but no change in the angle of deviation or symptoms occurred. A base-in prism was used unsuccessfully in Case No. 6 to remove diplopia.

Table 1 itemizes patient data, that is, age, sex, preoperative deviations, binocularity, surgeries performed, and postoperative alignments and binocularity at the last follow-up. All patients underwent full ophthalmologic and orthoptic evaluations.
Deviations were measured using prism and alternating cover tests, while patients fixated on accommodation controlling targets at 6 and $\frac{1}{3} \mathrm{~m}$. An additional near measurement was obtained $1 \mathrm{~h}$ after monocular occlusion of the habitually deviating eye. All measurements were performed with normal optical correction in place, if prescribed. Myopia $>1.00 \mathrm{D}$, astigmatism $>1.50 \mathrm{D}$, and hyperopia $>3.00 \mathrm{D}$ were usually treated with glasses that fully corrected myopia or astigmatism, and cycloplegic hyperopic refraction to $1.00-1.50 \mathrm{D}$ less than full before a final surgical decision was made.

Abnormalities in duction and version were checked and graded on a scale of 0 (normal) to -4 (inability to move into the field of action). Stereopsis was evaluated using the Titmus stereotest at near and fusion, using the Worth four-dot test. Surgery was recommended if manifest tropia was present more than 50\% of the time as determined either by ocular examinations or if there was a deterioration of exotropia in terms of frequency or magnitude. We explained to parents before operation that alternate patching or prism therapy would be required in cases of postoperative overcorrection at distance and/or at near.

Slanted bilateral MR resection was performed in all patients. The upper edge of the MR was resected according to the distance exodeviation and the lower edge of the MR was resected according to near exodeviation after a suture had been threaded obliquely with bias across the muscle (Figure 1, upper). Thus, the lower edge was resected more than the upper edge. Following resection, the MR was reattached at its original insertion (Figure 1, lower).

Postoperative follow-up intervals were determined according to patient status, but examinations were usually scheduled at 1 day, 1 week, and at 1, 3, 6, and 12

Table 1 Database for 10 patients treated

\begin{tabular}{|c|c|c|c|c|c|c|c|c|c|}
\hline \multirow[t]{2}{*}{ Case no. } & \multirow{2}{*}{$\begin{array}{c}\text { Age } \\
\text { (years)/sex }\end{array}$} & \multicolumn{3}{|c|}{ Preoperative results } & \multirow{2}{*}{$\begin{array}{c}M R \\
\text { Resection } \\
(m m) U P / L P\end{array}$} & \multicolumn{3}{|c|}{ Postoperative results } & \multirow{2}{*}{$\begin{array}{c}\text { Follow-up } \\
\quad(\mathrm{mo})\end{array}$} \\
\hline & & $\begin{array}{c}X(T) \text { pattern } \\
D / N(P D)\end{array}$ & $\begin{array}{l}\text { Difference, } \\
N-D(P D)\end{array}$ & $\begin{array}{l}\text { Stereopsis } \mathrm{a} \\
\quad \text { fusion }\end{array}$ & & $\begin{array}{l}X(T) \text { pattern } \\
D / N(P D)\end{array}$ & $\begin{array}{c}\text { Difference, } \\
N-D(P D)\end{array}$ & $\begin{array}{l}\text { Stereopsis/ } \\
\text { fusion }\end{array}$ & \\
\hline 1 & $5 / \mathrm{M}$ & $25 / 35$ & 10 & Fly / - & $5 / 6$ & $20 / 35$ & 15 & $800 /-$ & 58 \\
\hline 2 & $12 / \mathrm{F}$ & $25 / 38$ & 13 & $200 /-$ & $5 / 6$ & $20 / 25$ & 5 & $140 /+$ & 61 \\
\hline 3 & $10 / \mathrm{F}$ & $25 / 35$ & 10 & $200 /+$ & $5 / 6$ & $20 / 30$ & 10 & $60 /+$ & 6 \\
\hline 4 & $12 / \mathrm{M}$ & $20 / 30$ & 10 & $50 /+$ & $4 / 5.5$ & $10 / 20$ & 10 & $80 /-$ & 62 \\
\hline 5 & $9 / \mathrm{M}$ & $20 / 33$ & 13 & $50 /-$ & $4 / 5.5$ & $15 / 20$ & 5 & $40 /-$ & 59 \\
\hline 6 & $17 / \mathrm{M}$ & $15 / 25$ & 10 & $50 /+$ & $4 / 5$ & $10 / 14$ & 4 & $80 /+$ & 6 \\
\hline 7 & $10 / \mathrm{M}$ & $25 / 43$ & 18 & $400 /-$ & $5 / 6.5$ & $25 / 35$ & 10 & $200 /-$ & 36 \\
\hline 8 & $9 / F$ & $20 / 30$ & 10 & $-/-$ & $4 / 5.5$ & $20 / 25$ & 5 & $80 /-$ & 51 \\
\hline 9 & $9 / \mathrm{F}$ & $15 / 25$ & 10 & $40 /+$ & $3 / 5$ & $12 / 23$ & 11 & $40 /+$ & 36 \\
\hline 10 & $10 / \mathrm{M}$ & $40 / 50$ & 10 & $400 /-$ & $6.5 / 7.5$ & $11 / 19$ & 8 & $400 /-$ & 12 \\
\hline
\end{tabular}

${ }^{\mathrm{a}}$ Seconds of arc. $\mathrm{X}(\mathrm{T})=$ exotropia; $\mathrm{D} / \mathrm{N}=$ distance $/$ near; $\mathrm{N}-\mathrm{D}=$ near minus distance; $\mathrm{MR}=$ medial rectus; $\mathrm{UP} / \mathrm{LP}=$ upper pole $/$ lower pole; $\mathrm{PD}=$ prism diopters; $-=$ no existence of fusion; $\mathrm{mo}=$ months. 


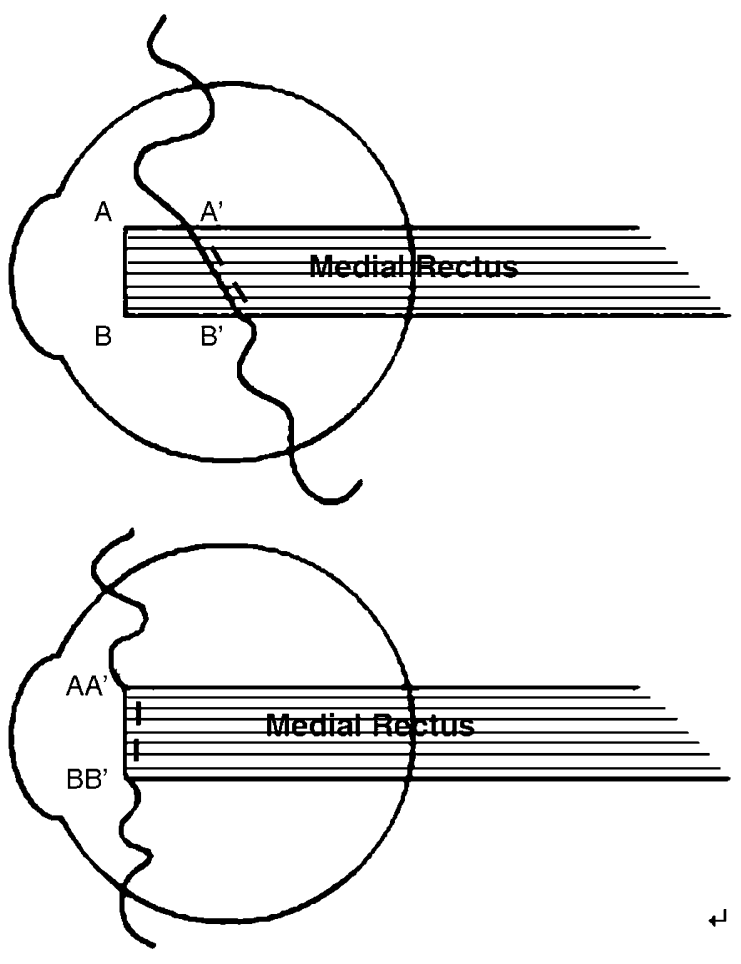

Figure 1 Diagram of slanted MR resection. The suture was threaded obliquely, on a bias (upper). After the lower muscle horn had been resected more than the upper horn, the muscle was reattached to its original insertion (lower). (A) Upper pole of original insertion of MR and (B) lower pole of original insertion of MR. $\mathrm{AA}^{\prime}$ means reattachment at upper pole of original insertion of $\mathrm{MR}$ after resection. $\mathrm{BB}^{\prime}$ means reattachment at lower pole of original insertion of MR after resection.

months after surgery, and annually thereafter. Postoperatively, alternate patching was performed in cases that manifested a consecutive esotropia with diplopia at distance and/or near. Alternate patching was discontinued when a patient maintained single binocular vision.

A successful outcome was defined as a postoperative residual deviation at near and distance $\leq 10 \mathrm{PD}$ and a near-distance exodeviation difference $\leq 10 \mathrm{PD}$.

The paired $t$-test was used to compare: mean distance angle of deviation preoperatively and postoperatively; mean near angle of deviation preoperatively and postoperatively; and mean near-distance exodeviation difference preoperatively and postoperatively. In addition, mean postoperative deviated angles at each scheduled follow-up were calculated and compared using the paired $t$-test. Microsoft Excel (version 5.00, 2002) was used for all statistical calculations.

The impact of slanted resection on the collapse of neardistance difference was calculated, specifically, the mean near-distance difference of exodeviation before and after surgery was divided by the mean amount of slanted resection, which was defined as the mean difference
Table 2 Postoperative change of deviated angles in treated children

\begin{tabular}{lrrrrr}
\hline Postoperative & \multirow{2}{*}{$\begin{array}{c}\text { No. of } \\
\text { cases }\end{array}$} & \multicolumn{4}{c}{ Deviated angle (PD, mean $\pm S D)$} \\
\cline { 3 - 6 } & & Distance & P-value* & Near & P-value* \\
\hline 1 day & 10 & $-4.1 \pm 10.1$ & & $3.4 \pm 9.1$ & \\
1 week & 10 & $-0.2 \pm 5.1$ & 0.289 & $5.5 \pm 4.6$ & 0.521 \\
1 month & 10 & $3.8 \pm 3.2$ & 0.050 & $7.8 \pm 5.9$ & 0.352 \\
3 months & 9 & $9.9 \pm 3.3$ & 0.000 & $17.8 \pm 5.7$ & 0.002 \\
6 months & 9 & $14.2 \pm 3.8$ & 0.019 & $22.1 \pm 5.4$ & 0.012 \\
1 year & 7 & $15.4 \pm 3.5$ & 0.523 & $22.4 \pm 3.4$ & 0.918 \\
Last visit & 10 & $16.3 \pm 5.4$ & 0.712 & $24.6 \pm 6.9$ & 0.444 \\
\hline
\end{tabular}

${ }^{*} P$-value between the previous and the current angles at each time. $-=$ esodeviation.

between the upper and lower poles of the slanted resection.

\section{Results}

The postoperative alignments and binocularity at the last follow-up visit, and the duration of follow-up for the 10 patients are listed in Table 1. Before surgery, the mean exodeviation at distance was $23.0 \mathrm{PD}$ (range, 15-40 PD) and at near, $34.3 \mathrm{PD}$ (range, 25-50 PD). At the last followup, the mean exodeviation at distance was 16.3 PD (range, 10-25 PD) and at near, 24.6 PD (range, 14-35 PD). Thus, significant postoperative reductions were achieved in exodeviation at distance $(P=0.029)$, as well as at near $(P=0.009)$. Near-distance differences were reduced from a preoperative mean of $11.4 \mathrm{PD}$ (range, 10-18 PD) to a postoperative mean of $8.3 \mathrm{PD}$ (range, 5-15 PD), which was statistically significant $(P=0.039)$.

On postoperative day 1 , no patient showed duction limitation in the operated eye or a torsional problem. However, five of the 10 patients (50\%) had esodeviations at distance and/or at near ranging from 4 to $25 \mathrm{PD}$. Two patients with esodeviations of 4 and $6 \mathrm{PD}$, respectively, at distance had no diplopia. Others $(n=3)$ with diplopia performed alternate patching. Two of these three patients resolved at 1 and 2 weeks postoperatively. One patient (No. 10) had 25 PD of esotropia at distance and $15 \mathrm{PD}$ at near with diplopia on postoperative day 1 . This patient was able to discontinue alternate patching 1 month after the operation.

The greatest amounts of exodrift observed during the postoperative 6-month period are shown in Table 2.

Thereafter, no statistical significance was found between mean deviated angles at far or near.

At the final follow-up examination, no patient met the criteria of surgical success; all had recurrent exotropia. However, near-distance differences had collapsed in four of the 10 patients. A 1-mm difference between the upper 
and lower poles of the bimedial recti slanted resection reduced near-distance difference by 2.0 PD.

Stereopsis at near was improved postoperatively rather than preoperatively in six patients. One of six patients who preoperatively lost fusional ability achieved fusion at distance or at near postoperatively.

\section{Discussion}

Exotropia with convergence insufficiency is less frequent than other forms of exodeviation. ${ }^{3,4}$ Several surgical procedures in this type of exotropia have been modified because results were unsatisfactory in exotropia with convergence insufficiency. ${ }^{1,2,7-12}$

Kraft et $a^{1}$ described a technique wherein monocular strengthening of MR resection exceeds the LR weakening. In all patients, near deviation was corrected and near-distance difference was significantly reduced. However, adjustable surgery was performed in five of 14 patients in their study, and thus the results obtained are subject to adjustable surgery-associated bias.

The results of slanted MR resection have been retrospectively studied in only six patients with convergence insufficiency type exotropia in the literature. ${ }^{8,9}$ All six patients showed postoperative alignment within 10 PD in all field gazes without diplopia. In contrast, no patient was treated successfully in the present study. This difference could be due to several factors. First, preoperative deviated angle degree may have affect surgical results. In the previous reports, patients had 2-10 PD of exophoria at distance, ${ }^{8,9}$ whereas in the present study, the minimal angle of exotropia was 15 PD at distance with a mean of 23 PD. Second, it has been reported that $X(T)$ shows a strong tendency to recur and drift into permanent exotropia with time after surgery. ${ }^{13-18}$ Postoperative success rates as quoted by previous reports vary from 41 to $95 \%$, which may be explained by different follow-up periods. A general drift in postoperative alignment with time was also demonstrated by the present study (Table 2); moreover, the follow-up period of the present study was much longer than of previous reports. ${ }^{8,9}$ Third, Choi et al ${ }^{7}$ stated that the effect of slanting, as predicted by mathematical models, is negligible and that muscle fibres probably rearrange themselves to negate any effect of slanting.

Snir et $a l^{2}$ concluded that slanted recession of the LR was superior to standard recession at reducing both distance and near exodeviation and at collapsing neardistance differences. They found that a 1-mm difference between upper and lower poles of the slanted recession reduced the near-distance difference by $4.6 \mathrm{PD}$. To date, no study has calculated the impact of slanted resection on collapse of near-distance differences, with the exception of the present study. On comparing the data of
Snir et $a l^{2}$ with that of the present study, it appears that the slanted MR procedure may be less effective than the slanted LR recession procedure at reducing near exodeviation and collapsing near-distance differences. These results suggest that the amount of slanting in the present study may be too small to correct the neardistance differences successfully and there still remains a possibility for an increased slanting to make any significant difference. The relatively short (12 months) duration of follow-up in their study may be one of cause of these surgical result differences (39 months).

Therefore, a controlled comparison between slanted LR recession and slanted MR resection is probably warranted.

No report has been issued to date about postoperative torsion or $\mathrm{A}$ or $\mathrm{V}$ phenomenon associated with the slanted procedure..$^{2,8,9,19,20}$ In a study on A-V pattern strabismus by Boyd et al, ${ }^{19}$ each $1 \mathrm{~mm}$ of slanting recession or resection of the muscle margins produced a horizontal deviation change of 3 PD between up and down gaze. Only 1-2 mm slanting procedure in the present study resulted in no postoperative $\mathrm{A}$ or $\mathrm{V}$ phenomenon.

Several authors have reported on postoperative diplopia in convergence insufficiency exotropia. Hermann ${ }^{10}$ reported on 14 patients treated by bilateral MR resection. Fresnel prisms were used postoperatively in all of these patients, and time to esotropia and diplopia resolution at distance averaged 2.7 months. von Noorden ${ }^{11}$ reported on six patients who received bilateral MR resections; Fresnel prisms were required to treat diplopia at distance in five patients for 5 weeks, and in one patient for 5 months. Choi et $a l^{7}$ performed unilateral or bilateral MR resection(s) with an adjustable suture and target angles of 10-20 PD esotropia at distance and 510 PD esotropia at near; postoperatively Fresnel prisms were used in 14 of their 21 cases. Of the 14 cases, 11 were able to discontinue prism use at 3 months after surgery, whereas the other three cases required prisms for 6 months or over. However, although the above reports address postoperative diplopia frequency, they make no mention of postoperative esotropia for slanted MR resection in convergence insufficiency type $X(T) ., 8$ Nemet and Stolovitch ${ }^{8}$ described that esotropia at distance tends to diminish and disappear with time, and that it ultimately results in convergence insufficiency recurrence. The present study also demonstrated that postoperative esotropia or diplopia was short-lived (less than 4 weeks) and that the postoperative angle had tendency to exodrift with time.

In conclusion, although slanted bilateral MR resection has little risk of creating long-term postoperative esodeviation at distance in $\mathrm{X}(\mathrm{T})$ with near-distance exodeviation differences of $>10$ PD, this method shows 
unsatisfactory results in terms of reducing exodeviation and collapsing near-distance differences after long-term follow-up.

\section{References}

1 Kraft SP, Levin AV, Enzenauer RW. Unilateral surgery for exotropia with convergence weakness. J Pediatr Ophthalmol Strab 1995; 32: 183-187.

2 Snir M, Axer-Siegel R, Shalev B, Sherf I, Yassur Y. Slanted lateral rectus recession for exotropia with convergence weakness. Ophthalmology 1999; 106: 992-996.

3 Kushner BJ, Morton GV. Distance/near differences in intermittent exotropia. Arch Ophthalmol 1998; 116: 478-486.

4 Burian HM. Exodeviations: their classification, diagnosis and treatment. Am J Ophthalmol 1966; 62: 1161-1166.

5 Daum KM. Convergence insufficiency. Am J Optom Physiol Opt 1984; 61: 16-22.

6 Birnbaum MH, Soden R, Cohen AH. Efficacy of vision therapy for convergence insufficiency in an adult male population. J Am Optom Assoc 1999; 70: 225-232.

7 Choi DG, Rosenbaum AL. Medial rectus resection(s) with adjustable suture for intermittent exotropia of the convergence insufficiency type. J AAPOS 2001; 5: 13-17.

8 Nemet $P$, Stolovitch C. Biased resection of the medial recti: a new surgical approach to convergence insufficiency. Binocul Vision Strab Q 1990; 5: 213-216.

9 Biedner B. Treatment of convergence insufficiency by single medial rectus muscle slanting resection. Ophthal Surg Lasers 1997; 28: 347-348.
10 Hermann JS. Surgical therapy for convergence insufficiency. J Pediatr Ophthalmol Strab 1981; 18: 28-31.

11 von Noorden GK. Resection of both medial rectus muscles in organic convergence insufficiency. Am J Ophthalmol 1976; 81: 223-226.

12 Haldi BA. Surgical management of convergence insufficiency. Am Orthoptic J 1978; 28: 106-109.

13 Scott WE, Keech R, Mash AJ. The postoperative results and stability of exodeviations. Arch Ophthalmol 1981; 99: 1814-1818.

14 Maruo T, Kubota N, Sakaue T, Usui C. Intermittent exotropia surgery in children: long term outcome regarding changes in binocular alignment. A study of 666 cases. Binocul Vision Strab Q 2001; 16: 265-270.

15 Stoller SH, Simon JW, Lininger LL. Bilateral lateral rectus recession for exotropia: a survival analysis. J Pediatr Ophthalmol Strab 1994; 31: 89-92.

16 Beneish R, Flanders M. The role of stereopsis and early postoperative alignment in long-term surgical results of intermittent exotropia. Can J Ophthalmol 1994; 29: 119-124.

17 Pratt-Johnson J, Wee HS. Suppression associated with exotropia. Can J Ophthalmol 1969; 4: 136-144.

18 Richard JM, Parks MM. Intermittent exotropia. Surgical results in different age groups. Ophthalmology 1983; 90: 1172-1177.

19 Boyd TA, Leitch GT, Budd GE. A new treatment for 'A' and ' $\mathrm{V}^{\prime}$ patterns in strabismus by slanting muscle insertions. A preliminary report. Can J Ophthalmol 1971; 6: 170-177.

20 Nemet P, Stolovich C, Loewenstein A. A new operation: slanted (reinsertion) medial rectus muscle recession surgery for convergence excess esotropia: results in 12 cases. Binocul Vision Strab Q 1993; 8: 241-246. 